\title{
LA POLITICA COME SCIENZA
}

di Giovanni Sartori

La filosofia non presuppone un metodo filosofico. Quantomeno, non esiste un metodo filosofico codificato. Al massimo si potrà dire che la filosofia presuppone un « corretto ragionare », e cioè la logica. Ma certo la logica non sta alla filosofia cosí come il metodo scientifico sta alla scienza. Sarebbe azzardato asserire che non c'è filosofia senza logica; e certo, molti illustri filosofi hanno ampiamente derogato dall'unica logica che la tradizione filosofica ha codificato: la logica Aristotelica. Per contro si sostiene che non c'è scienza propriamente detta senza metodo scientifico. Questo metodo scientifico non è immutabile, è uno ma anche plurimo, ed' è in continua evoluzione. Il che non toglie che la scienza presuppone un metodo scientifico. E in forza di questo criterio, difatti, che la nascita del pensiero scientifico e il suo distacco dal pensiero filosofico viene collocata nei secoli XVI-XVIII, nell'arco di tempo che va da Bacone a Galileo e, conclusivamente, a Newton ${ }^{1}$.

Lo spirito scientifico del XVII secolo costituisce un punto di riferimento obbligato anche per una storia delle scienze dell'uomo? Sí e no. Sí nella misura in cui nel XVII secolo si afferma il principio secondo il quale non c'è scienza senza metodo scientifico. No, nella misura in cui questo quadro di riferimento privilegia un solo metodo e fa coincidere il metodo scientifico con il « metodo Newtoniano ».

Ścienza è un singolare che sottintende un plurale, e cioè una pluralità di scienze. In primo luogo, si deve tener presente

La prima parte di questo articolo è apparsa con il titolo Cosa è 'Politica' nel n. 1, 1972, pp. 3-26.

1 Per la complessità della genesi delle scienze dalla tradizione filosofica, vedi spec. E. Cassirer, Storia della filosofia moderna, 4 voll., Torino, Einaudi, 1952-58. 
che la geometria e la matematica hanno fornito sin dall'antichità un primo modello e il primo archetipo della scientificità ${ }^{2}$. In secondo luogo, occorre ricordare che le scienze naturali (al plurale) precedono di gran lunga la fisica di Newton e che non si sono mai riconosciute in quel modello. La botanica, la mineralogia, la zoologia e, in parte, la biologia e la medicina sono, pregiudizialmente, scienze classificatorie. Si deve tener presente, pertanto, che esiste una accezione lata di scienza che sfugge ad ogni riduzione unitaria. Se la fisica pone un modello che oggi diciamo "fisicalista », esistono molte scienze che non sono riducibili a quel modello. Ne consegue che il metodo scientifico che pone in essere una scienza non è necessariamente quello del fisicalismo.

Si deve distinguere, pertanto, tra scienza in senso stretto e scienza in senso lato. Nell'accezione stretta, tutte le scienze vengono commisurate ad una scienza regina che ne costituisce l'archetipo: qui «scienza » sta per dire, in sostanza, scienza esatta, scienza di tipo fisicalista. Nell'accezione lata, l'unità della scienza è riferita al minimo comun denominatore di qualsiasi discorso scientifico: qui «scienza » sta per scienza in generale. In questo secondo caso noi riconosciamo una pluralità di scienze e di metodi scientifici che vanno - con tutta una gamma di casi intermedi - dalle scienze «classificatorie » alle scienze « fisicaliste». E questa concezione flessibile e poliedrica quella che meglio apre e consente il discorso sulle scienze dell'uomo ${ }^{3}$.

Non basta. Nel concepire la scienza con flessibilità il metro storiografico risulta necessariamente piú elastico del metro sta-

2 Cf. L. Brunschwicg, Les étapes de la philosopbie mathématique, Paris, Presses Universitaires de France, 1912; e H. Weyl, Philosophy of Mathematics and Natural Science, Princeton, Princeton University Press, 1949.

${ }_{3} \mathrm{Gli}$ epistemologi contemporanei si possono classificare a seconda della maggiore o minore aderenza al modello fisicalista. In quest'ordine mi limito a richiamare i nomi di Rudolph Carnap, Carl G. Hempel, Ernst Nagel, Karl Popper e, all'estremo opposto di Carnap, Michael Polanyi. A titolo introduttivo è illuminante l'agile libro di T.S. Kuhn, The Structure of Scientific Revolutions, Chicago, University of Chicago Press, 1952, tr. it. La struttura delle rivoluzioni scientifiche, Torino, Einaudi, 1969. In chiave metodologica il migliore testo specifico è A. Kaplan, The Conduct of Enquiry: Methodology for Bebavioral Science, San Francisco, Chandler, 1964. Una utile collezione antologica è M. Brodbeck (ed.), Readings in the Philosopby of the Social Sciences, Toronto, Macmillan, 1968. 
bilito dall'epistemologia contemporanea. Quel che può risultare scienza in riferimento al passato, e cioè in una prospettiva diacronica, non è detto che possa essere considerato scienza al presente, nella prospettiva del nostro tempo. Distinguendo tra questi due passi o metri di flessibilità si sciolgono molte futili polemiche. Aristotile e Machiavelli furono «scienziati » della politica? In sede storiografica si può rispondere affermativamente; in sede epistemologica si deve rispondere negativamente. Lo storico potrà osservare che una « osservazione realistica » costituisce la premessa, e resta parte integrante, della forma mentis scientifica. Potrà anche rilevare che Aristotile non si colloca in una storia della scienza politica (e di altre scienze ancora) semplicemente a titolo di attento descrittore delle vicende del suo tempo, ma specificamente per la sua forma mentis classificatoria. Del pari lo storico potrà cogliere la « scientificità » di Machiavelli nel fatto che con lui l'osservatore si distacca - pur non spogliandosi dei propri fini e valori - dalle cose osservate; e anche rilevando che a questo modo Machiavelli rompe con la tradizione filosofica, e cioè si distacca della filosofia. Tutto vero. Ma l'epistemologo ha il diritto - e anche il dovere - di ribattere che se l'osservazione realistica prelude alla scienza, di per sé presa non è ancora scienza. Analogamente l'epistemologo dovrà precisare che se la scienza non è filosofia, non si fa scienza semplicemente perché non si fa filosofia.

La differenza tra il metro di giudizio storico e storiografico da un lato, e il metro di giudizio epistemologico dall'altro, si ripropone anche per autori che ci sono vicinissimi nel tempo: Gaetano Mosca (1858-1941), per citare un nome che tutti associano alla scienza politica. Qual è la scientificità dei suoi Elementi di scienza politica (1895 e 1922)? Il metodo di Mosca era storico-induttivo: un metodo, se si vuole, empirico ma non scientifico. L'epistemologo dirà, pertanto, che la scienza politica di Mosca era pre-scientifica. Fatte le debite differenze, Mosca si qualifica come politologo alla stregua di Machiavelli, per il suo « realismo » e per il fatto di essere uno «specialista » il quale, in quanto tale, riafferma l'esigenza di uno studio autonomo della politica; nel caso di Mosca, autonomo dalla scienza giuridica, e specificamente dalla costituzionalizzazione della politica. Per il nostro metro epistemologico, dunque, Mosca è ancora da classificare nella fase pre-scientifica della scienza 
politica ${ }^{4}$. Lo stesso vale per Roberto Michels. Semmai chi si qualifica, dal punto di vista della scientificità, è Vilfredo $\mathrm{Pa}$ reto, anche se il suo metodo scientifico risulta ai nostri occhi alquanto primitivo ed impuro ${ }^{5}$. Eppure basta riassumere la prospettiva storica per avvertire che il metro epistemologico taglia troppo facilmente la testa al toro. Nel caso in esame un fatto è certo: a dispetto della loro insoddisfacente scientificità, Mosca, Pareto e Michels hanno pur sempre ipotizzato e teorizzato tre « leggi » della politica - rispettivamente la legge della classe politica, la legge della circolazione delle élites, e la legge di ferro dell'oligarchia - le quali sono ancor oggi al centro del dibattito politologico. Domanda: la formulazione di « leggi » non è forse un obiettivo, e non dei minori, della conoscenza che diciamo scientifica? A questa prima perplessità se ne aggiunge una seconda. $\mathrm{Va}$ bene che il metodo storico-induttivo non è scientifico; ma la scienza politica può davvero ignorare la storia e l'esperienza storica? Oppure il metodo della scienza politica, pur essendo scientifico e non storico, deve includere

${ }^{4}$ La discussione su Mosca è sempre attuale. Tra gli scritti piú recenti segnalo: A. Passerin d'Entrèves, Gaetano Mosca e la libertà (1959), ora accolto nel vol. Obbedienza e resistenza in una società democratica, Milano, Comunità, 1970; N. Bobbio, Gaetano Mosca e la scienza politica (1960), e Gaetano Mosca e la teoria della classe politica (1962), entrambi accolti nel suo vol. Saggi sulla scienza politica in Italia, Bari, Laterza, 1969, nonché la sua Introduzione alla ed. ridotta degli Elementi di scienza politica (intitolata: G. Mosca, La classe politica), Bari, Laterza, 1966; M. Fotia, Classe politica, liberalismo, democrazia in G. Mosca, in «Rivista di Sociologia », IV (1966), pp. 5-68; F. Vecchini, La pensée politique de $G$. Mosca et ses différentes adaptations au cours du $X X^{\circ}$ siècle en Italie, Paris, Cujas, 1968; L. Cavalli, «Parte Terza: Gaetano Mosca », in Il mutamento sociale, Bologna, Il Mulino, 1970; A. Lombardo, Sociologia e scienza politica in G. Mosca, in « Rivista Italiana di Scienza Politica », I (1971), pp. 297-323.

${ }_{5}$ Su Pareto vedi i cinque scritti ora raccolti in N. Bobbio, Saggi sulla scienza politica in Italia, cit.; e per la bibliografia piú recente G. Busino, Storia, economia, sociologia e politica nelle ricerche recenti sull'opera di V. Pareto, in «Rivista Storica Italiana », LXXX (1968), pp. 938-963. Busino è il curatore delle Oeuvres complètes de V. Pareto, e della rivista Cabiers Vilfredo Pareto, di cui vedi spec. i n.ri 1 (1963), 5 (1965), 12 (1967). Assai fine il cap. su Pareto di R. Aron, Les étapes de la pensée sociologique, Paris, Gallimard, 1967. Quanto a Michels, la migliore interpretazione critica è la Introduzione di Juan Linz alla nuova ed. della Sociologia del partito politico, Bologna, Il Mulino, 1966, fondata sulla ult. ed. tedesca, del 1925, del vol. michelsiano. 
un modo di «trattare», sia pure ai propri fini, l'esperienza storica ${ }^{6}$ ?

Anche se alla fine dovremo commisurare la scienza politica degli anni sessanta secondo $\mathrm{i}$ propri principii - e cioè con $\mathrm{i}$ criteri di scientificità stabiliti dall'epistemologo - in attesa di arrivare alla fase piú recente della disciplina, si deve sottolineare che per circa un secolo si è parlato di «scienza politica » - non senza merito e ragione - per qualificare l'incontro tra un modo autonomo di studiare la politica (autonomia a parte subiecti) e una politica vista nella propria autonomia (autonomia a parte obiecti). Un modo autonomo di studiare la politica nel senso che il politologo non è un filosofo, non è un giurista, non è un economista e non è un sociologo. Una politica vista nella propria autonomia nel senso illustrato nella prima parte: per dire che la politica ha i suoi imperativi, le sue « leggi », e che non è riducibile ad altro ${ }^{7}$.

A dispetto dei canoni della scientificità, il grosso del nostro sapere in materia politica risale ancora all' $\ll$ incontro significativo » tra l'autonomia dell'osservatore politico e l'autonomia della politica che osserva. Dico a dispetto dei canoni della scientificità perché questo non è, a rigore, un incontro tra «scienza » e «politica ». Ma la lezione che ci proviene dalla storia della scienza e delle sue varie accezioni è, appunto, quanti incontri sono mancati oppure si sono rivelati infecondi. Oggi stiamo ritentando l'incontro tra matematica e politica: eppure non è ancora detto che le nostre matematiche siano arrivate a uno sviluppo che consenta un incontro fecondo. La matematica costituisce da millenni un modello e uno strumento di scientificità: eppure quei filosofi che furono grandi matematici non hanno apportato nulla alle nostre conoscenze politiche. Ancora: lo « spirito scientifico » di Galileo e di Newton pervade e carattenizza l'età dei lumi. Si è già detto di Hobbes. Ma si prendano gli Enciclopedisti, oppure il materialismo sensistico e mec-

6 Il ritorno alla storia del politologo costituisce il motivo di fondo del contributo di N. Matteucci, La scienza politica, in AA. VV., Le scienze umane in Italia oggi, Bologna, Il Mulino, 1971, pp. 219-58. Mentre condivido lo spirito del richiamo di Matteucci, resta la differenza che al politologo non spetta di trattare la storia al modo dello storico. D'altronde anche lo storico ha i suoi problemi. Vedili minuziosamente elencati nella caccia agli errori di D. H. Fischer, Historians' Fallacies, New York, Harper \& Row, 1970. Cf. anche n. 24 infra.

${ }^{7}$ Cosa è «politica», cit., spec. pp. 10-17, 24-26. 
canico di Condillac (1714-1780), di La Mettrie (1709-1751) e del barone d'Holbach (1723-1789). Gli Enciclopedisti, e ancor piú gli autori sunnominati, hanno indubbiamente, anche se variamente, applicato all'uomo e alla politica la visione scientifica dell'universo del loro tempo. E dunque nel settecento un incontro tra scienza e politica c'è stato: ma i frutti sono stati scarsi. Gli autori di quel secolo che leggiamo con ben altro profitto sono Hume e Burke, Montesquieu e l'antiscientifico Rousseau.

Non si pecca di lassismo, allora, quando si ricollega la scienza politica non tanto alla «scientificità intrinseca » della disciplina - scienza politica in senso stretto - quanto alla « autonomia » del politologo - scienza politica in senso lato e cioè al suo distacco da tutti quei modi di conoscere la politica che si rivelano ricoperti e filtrati da occhiali speculativi, etici, giuridici, sociologici e quant'altro. I distacchi sono parecchi, come si vede. Ma quello decisivo è il distacco dalla filosofia. E questo, difatti, il distacco che pone in essere una scienza politica nel senso lato della dizione.

\section{Filosofia, scienza e teoria}

Se tutte le scienze nascono distaccandosi dalla filosofia, taluni distacchi sono ormai acquisiti. Il cultore delle scienze naturali e sperimentali non sente piú il bisogno di definire se stesso come non-filosofo, nella propria opposizione-differenza dalla filosofia. Diverso è il caso delle scienze dell'uomo, il cui distacco è recente e anche incompiuto. Dal che consegue che per le scienze dell'uomo il problema dei rapporti con la filosofia resta un problema aperto.

Dati due termini - filosofia e scienza - da specificare $a$ contrario, o per differenza, la strategia ottimale è di ricavare il termine meno noto dal termine meglio noto. Nel caso delle scienze fisiche, per esempio, conviene partire da «scienza» per ricavarne una identificazione negativa di filosofia come nonscienza. Ma nel caso delle scienze dell'uomo conviene rispettare l'ordine genetico e partire da «filosofia» per ricavarne una identificazione negativa di scienza come non-filosofia. Con questo non si vuol dire che si fa scienza semplicemente per difetto di filosofia. Per quanto si voglia intendere la nozione di scienza con la massima latitudine, non giova farne una nozione pura- 
mente residuale. Asserire che la scienza non è filosofia è cogliere il « distacco » della prima dalla seconda nella consecutio storica in cui è avvenuto: partendo dalla filosofia per arrivare alla scienza.

Il quesito generale è che cosa sia la filosofia nella sua differenza dalla scienza. Il quesito specifico è che cosa differenzia la filosofia (della) politica dalla scienza (della) politica. Il secondo quesito è ovviamente ricompreso nel primo; ma pone anche dei problemi sui generis.

La filosofia può essere vista come un contenuto di sapere e/o come un metodo di acquisizione di quel sapere. E vale partire dalla individuazione dei contenuti ricorrenti e caratterizzanti del filosofare. E la via seguita di recente da Norberto Bobbio quando riconduce la filosofia politica a quattro grandi temi di riflessione: 1) ricerca della miglior forma di governo o dell'ottima repubblica, 2) ricerca del fondamento dello Stato e giustificazione dell'obbligo politico, 3) ricerca della natura della politica, o meglio, dell'essenza della politicità, 4) analisi del linguaggio politico ${ }^{8}$. Lasciando da parte l'ultima forma di filosofia politica, che è ancora la piú informe, non si può dubitare che le sue indicazioni sostantive siano chiarificatrici ai fini della individuazione della fattispecie. $\mathrm{Ma}$ il discorso non può finire qui.

Se $\mathrm{i}$ temi del filosofo sono diversi dai temi del politologo è perché l'uno guarda dove l'altro non vede, e cioè perché $\mathrm{i}$ criteri e gli obiettivi del primo non sono quelli del secondo. Lo spartiacque sta dunque nel «trattamento »e, in questo senso, nel metodo. Sempre seguendo la traccia di Bobbio il trattamento filosofico è caratterizzato da « almeno uno » dei seguenti elementi: 1) un criterio di verità che non è la verificazione ma semmai la coerenza deduttiva; 2) un intento che non è la spiegazione ma piuttosto la giustificazione; 3) la valutazione come presupposto e come scopo. Per il primo rispetto il trattamento filosofico non è empirico; per il secondo si caratterizza

8 I richiami a Bobbio si riferiscono a due scritti che si completano l'uno con 1'altro: Dei possibili rapporti tra filosofia politica e scienza politica, nel vol. coll. Tradizione e novità della filosofia della politica, Quaderni degli Annali della Facoltà di Giurisprudenza, Bari, 1971; e Considerazioni sulla filosofia politica, in « Rivista Italiana di Scienza Politica », I (1971), pp. 367-379. Nel vol. Tradizione e novità della filosofia politica vedi anche le relazioni di A. Passerin d'Entrèves, S. Cotta e V. Frosini. 
come normativo o prescrittivo; e per il terzo si precisa come un trattamento valutativo e assiologico ${ }^{9}$. Distinguendo tre elementi caratterizzanti, e proteggendosi con la clausola che ne basta uno, Bobbio supera la difficoltà presentata dalla grandissima varietà del filosofare. Se la scienza si divide in una pluralità di scienze, questa pluralità è una pluralità ordinata o comunque ordinabile. La filosofia si suddivide anch'essa, in concreto, in una pluralità di filosofie: ma questa pluralità va davvero in ordine sparso, in un grande e sfuggente disordine. Alcune filosofie sono altamente rarefatte, e cioè altamente speculative o, alla lettera, «metafisiche »; ma altre filosofie sono intrise di corpulenza empirica. Vi è un filosofare che è rigorosamente logico e deduttivo; ma vi è anche un filosofare che è « poesia », tutto fondato su metafore, assonanze e licenze che sono davvero poetiche. È vero che il filosofo è di solito valutativo e assiologico; ma nulla vieta al filosofo di teorizzare e praticare l'avalutatività.

L'impostazione di Bobbio supera - ripeto - questa difficoltà. Presenta anche il vantaggio di allineare i criteri costitutivi del trattamento filosofico a quelli del metodo scientifico, che ne sono il rovescio, e che pertanto consistono: 1) nel principio di verificazione, 2) nella spiegazione, 3) nella avalutatività ${ }^{10}$. Nondimeno alcuni problemi sussistono. In primo luogo la corrispondenza tra tematica (contenuto) e trattamento (metodo) non è sempre convincente. Bobbio consente che $\mathrm{Ma}$ chiavelli sia assegnato alla filosofia in forza del suo tema: l'indagine sulla natura della politica. Ma è arduo confortare questa assegnazione in base a uno qualsiasi dei tre criteri che contraddistinguono, per Bobbio, il filosofare. Per questo rispetto Machiavelli è piú vicino alla verificazione che alla deduzione, alla spiegazione che alla giustificazione, e alla avalutatività che non alla assiologia. In secondo luogo non è chiaro se per i criteri della conoscenza scientifica valga una clausola di reciprocità, e cioè se l'adempienza a una sola delle tre condizioni sopracitate sia condizione sufficiente di «scienza». Ad occhio e croce si direbbe di no; e questo difetto di simmetria apre diversi interrogativi. Si può sospettare, tra l'altro, che la lista dei criteri differenzianti non sia ancora a punto. passim.

9 Dei possibili rapporti tra flosofia politica e scienza politica, loc. cit., ${ }_{10}$ Considerazioni sulla filosofia politica, cit., pp. 370-71. 
Nel differenziare la filosofia dalla scienza i piú approdano a una contrapposizione dicotomica, a due voci. Una prima dicotomia - che anche Bobbio sottolinea piú di altre - contrappone la filosofia come discorso assiologico-normativo alla scienza come discorso descrittivo-avalutativo. $\mathrm{Ma}$ non tutti convengono sulla validità di questa antitesi ${ }^{11}$. Una seconda partizione sottolinea questa differenza: che la filosofia è tale in quanto «sistema filosofico », e cioè concezione universale che si rifà $a b$ imis fundamentis, laddove la scienza è segmentata, non richiede globalità, e tantomeno una sistemazione dei principii primi del tutto. Una terza antitesi si riallaccia invece alla differenza tra il carattere discreto e non cumulativo della speculazione filosofica rispetto alla cumulabilità e trasmissibilità del sapere scientifico. Una ulteriore contrapposizione è quella tra il filosofare come indagine metafisica su «essenze »- di quel che sta prima, sotto o sopra le cose visibili, i fenomeni o le apparenze - e la scienza come rilevazione di « esistenze », di cose che si vedono, toccano o altrimenti accertano con l'esperimento. Infine, un'ultima antitesi è tra la filosofia come sapere «non applicabile », e cioè non commisurato a problemi di applicazione, e la scienza come sapere non solo operazionale ma anche operativo.

Presa singolarmente nessuna delle demarcazioni considerate è esauriente. Ma le possiamo conglobare in una sindrome. In tal caso sotto la voce «filosofia » cade il pensare caratterizzato da piú di uno - anche se non da tutti - i seguenti sintomi: 1) deduzione logica, 2) giustificazione, 3) valutazione normativa, 4) universalità e fondamentalità, 5) metafisica di essenze, 6) inapplicabilità. Per contro sotto la voce « scienza » cadrebbe il pensare caratterizzato da piú di uno - anche se non da tutti - i seguenti connotati: 1) verifica empirica, 2) spiegazione descrittiva, 3) avalutatività, 4) particolarità e cumulabilità, 5) rilevazioni di esistenze, 6) operazionalità e operatività.

A questo modo, comunque, abbiamo solo allungata l'enumerazione di Bobbio; il che rende simmetrica e piú elastica la clausola dei requisiti necessari e sufficienti (che diventa « piú

1 Per una conciliazione giudiziosa, cf. R. J. Pennock, Political Pbilosophy and Political Science, in O. Garceau (ed.), Political Research and Political Theory, Cambridge, Harvard University Press, 1968. Ma vedi meglio infra, e n. 30 . 
di uno », anche se «meno di tutti »). Per orientarsi può bastare. Ma continua a mancare un filo conduttore, un manico. Restano inevase due domande. In primo luogo, se esista un minimo comun denominatore che consenta di ricondurre la molteplicità delle filosofie alla unità di un medesimo filosofare. Inoltre, se è vero che il trattamento filosofico produce esiti (contenuti) cosí diversi dal trattamento scientifico, qual è se c'è - il fundamentum divisionis?

Prima di rispondere occorre sistemare la nomenclatura. Lo scibile non viene classificato soltanto sub specie di filosofia $o$ scienza: viene classificato anche sotto la voce «teoria ». Inoltre, in sede politica parliamo anche di «dottrine » e di «ideologie » che risultano diverse da pure e semplici « opinioni ». Dal che consegue che dobbiamo fermare, in via pregiudiziale, l'intero grappolo dei concetti che scompongono e qualificano lo scibile. Se non ci intendiamo su tutto il grappolo, il discorso si ingarbuglia prima di cominoiare. Basta toccare o spostare un tassello, e tutto il mosaico è da ricomporre. E certo molte controversie sono alimentate da malintesi sulla architettonica dell'insieme.

Tra tutte le voci sopra menzionate «teoria » è forse la piú polivalente e certo la prima da fissare. Etimologicamente theorein vuol dire vedere, e dunque teoria è "vista », visione. Non c’è nessuna particolare spiegazione del perché il concetto di teoria abbia mantenuto questa latitudine originaria mentre « scienza », che viene da scire, e che dunque aveva un significato altrettanto lato, ha finito per designare una conoscenza specializzata. Ma tant'è; e tanto vale rispettare la convenzione che fa di teoria il termine che attraversa tutto lo scibile. «Teoria » appartiene tanto alla filosofia (la teoria filosofica) quanto alla scienza (la teoria scientifica). Pertanto la dizione «teoria politica » non precisa se la teoria in questione sia filosofica o scientifica; precisa soltanto che si richiede un alto livello di elaborazione mentale. Una teoria potrà essere di natura filosofica o di natura scientifica; nondimeno la «statura teoretica » è capacità o talento di pochi. Se la denotazione di teoria è generalissima, la sua connotazione è aristocratica; la teoria sta sopra a cose che stanno sotto, a prodotti mentali di minore pregio $^{12}$.

${ }^{12}$ La nozione di teoria politica è tra le piú controverse. La complessità del problema risulta eo ipso dal lussureggiante A. Brecht, Political 
Quel che sta sotto alla teoria viene spesso detto, in sede politica, « dottrina ». Una dottrina politica ha minore rango intellettuale, o meglio euristico, di una teoria politica. Anche perché l'etichetta viene spesso riferita a proposte o programmi in ordine ai quali il fondamento teoretico importa meno del disegno concreto. $\mathrm{Ma}$ per quanto una dottrina politica non sia necessariamente da commisurare in chiave euristica, nondimeno un suo rango intellettuale lo possiede. Dal che consegue che anche la dottrina politica sta sopra a cose che le stanno sotto: per un verso le mere « opinioni », e per un altro verso l'« ideologia », entrambe caratterizzate da assenza di valore cognitivo. Vero è che il termine ideologia viene usato, nella tradizione marxista, non come una specie sottostante ma come una imputazione onnicomprendente ${ }^{13}$. In quest'ultima accezione tutto diventa ideologia, salvo la scienza quando è davvero scienza, e cioè quando non è scienza dichiarata borghese o capitalistica. $\mathrm{Ma}$ questa accezione esorbita dal problema considerato, che è

Theory: The Foundations of Twentietb-Century Political Thought, Princeton, Princeton University Press, 1959. Ma vedi, di Brecht, la voce Political Theory: Approaches, nella International Encyclopedia of the Social Sciences, New York, Macmillan \& Free Press, 1968 (seguita, ivi, dalla voce di Sheldon S. Wolin). Della vasta bibliografia segnalo: D. Easton, The Decline of Modern Political Theory, in « Journal of Politics », XIII (1951); T. D. Weldon, The Vocabulary of Politics: An Enquiry in the Making of Political Theories, London, Penguin, 1953; A. Cobban, The Decline of Political Theory, in "Political Science Quarterly », LXVIII (1953), n. 3, pp. 972-988; l'eccellente A. Rapoport, Various Meanings of 'Theory', in "American Political Science Review », LII (1958); L. Strauss, What is Political Pbilosopby?, Glencoe, Free Press, 1959; E. Weil, Philosophie politique, théorie politique, in « Revue Française de Science Politique », XI (1961), pp. 267-294 (fascicolo dedicato alla teoria politica); K. W. Deutsch e L. N. Rieselbach, Recent Trends in Political Theory and Political Pbilosopby, Philadelphia, Annals of the American Academy of Political and Social Science, July 1965; J. W. Chapman, Political Theory: Logical Structure and Enduring Types, nel vol. coll. L'idée de philosophie politique, Paris, Presses Universitaires de France, 1965; D. Germino, Beyond Ideology: The Revival of Political Theory, New York, Harper \& Row, 1967; R. Boudon, Notes sur la notion de théorie dans les sciences sociales, in «Archives Européennes de Sociologie », XI (1970), pp. 201-251. Vedi, infine, i tre voll. coll. della serie curata da P. Laslett (al quale si è aggiunto W. G. Runciman), Philosopby, Politics and Society, Oxford, Blackwell, rispettivamente del 1956, 1963 e 1967, che contiene ottimi contributi.

${ }_{13}$ Per i limiti di queste riduzioni (anche in riferimento alla sociologia della conoscenza) vedi, tra l'altro, J. N. Shklar, Political Theory and Ideology, New York, Macmillan, 1966, spec. l'Introduzione. 
di utilizzare le etichette disponibili al fine di una ordinata classificazione dello scibile. A questo fine serve invece l'accezione non-marxista, che si avvale di « ideologia » per designare il sottoprodotto semplificato ed emotivamente spendibile di talune filosofie o dottrine politiche.

Dalla presa in considerazione dell'intero grappolo si evince, in primo luogo, che la filosofia e la scienza sono configurabili come gli estremi di un continuum la cui zona intermedia sfugge ai due "tipi ideali » in questione; e che molto dipende, in secondo luogo, da questo dilemma: se ricomprendere senza residuo la teoria, a seconda dei casi, nella filosofia o nella scienza, oppure se mantenere la teoria come un tertium genus a sé stante. Va da sé che i connotati e la capienza della filosofia e della scienza mutano, e di parecchio, a seconda di come quel dilemma venga risolto. $E$ per risolverlo occorre chiarire un ultimo punto pregiudiziale: la differenza che passa tra l'incasellamento del già pensato, e il pensare in funzione di un incasellamento.

A detta di Benedetto Croce ogni storia è contemporanea: un passato veduto con gli occhi del presente. Il che non toglie che sia assurdo incasellare a forza, nella alternativa filosofia-oscienza, autori che ignoravano questa partizione. E utile, invece, un ricostruzione ex post, e cioè una storia del pensiero politico, intesa a classificare gli autori come filosofi $o$ no: intendendosi per non-filosofi coloro che non pretendevano di esserlo e che non attendevano alla costruzione di un «sistema ». E il caso di Machiavelli; ma è anche il caso, tra gli altri, di Burke, di Montesquieu, degli autori dei Federalist Papers, di Benjamin Constant e di Tocqueville. Ripeto: l'alternativa filosofia-o-scienza non è da applicare al passato; è una alternativa che ci poniamo oggi a futura memoria, sia per eliminare ibridi infecondi, sia per cercare la divisione del lavoro conoscitivo che meglio ci convenga.

La distinzione tra « retrospezione » e «prospezione », tra ricostruzione ex post e progettazione ex ante, consente anche di chiarire la collocazione della teoria. Retrospettivamente mi sembra indubbio che la teoria politica sia un tertium genus: quel genere che prepara e media il lungo passaggio dalla filosofia politica alla scienza politica strettamente intesa. Talché potremmo definire la teoria politica, nella sua irriducibilità, come il modo autonomo (né filosofico, né scientifico) di « vedere » la politica nella propria autonomia. Per quanto riguarda invece il futuro 
la teoria politica come terzo genere sembra destinata ad essere riassorbita. Di tanto una disciplina scientifica si consolida, di altrettanto sviluppa una teoria endogena, frutto della riflessione che la scienza compie su se stessa. Solo in via transitoria si ha una filosofia della scienza alla quale attendono i filosofi. In via definitiva sono $\mathrm{i}$ cultori della scienza «pura» che producono la teoria della propria scienza. E non c'è scienza compiuta che non sia, ad un tempo, scienza applicata e scienza teorica.

Fermiamo, riassumendo, tre punti. Primo: a tutt'oggi lungo il continuum i cui estremi sono segnati dai tipi ideali « filosofia » e «scienza » troviamo teorie politiche che non sono riconducibili né all'uno né all'altro ${ }^{14}$, anche se sono piú avvicinabili all'uno che all'altro. Secondo: in ogni caso tra la filosofia e la scienza politica resterà sempre una zona intermedia occupata, se non altro, da «dottrine politiche ». Terzo: teorie, dottrine e ideologie stanno tra di loro soprattutto in un ordine gerarchico che va da un massimo a un minimo di valore cognitivo e, all'inverso, da un minimo a un massimo di valore volontaristico. Deve essere chiaro, infine, che la dicotomia filosofia-scienza non ha validità retrospettiva ma prospettica. Se la retrodatiamo, occorre farlo con cautela e misura. Come diceva Leibniz, " on récule pour mieux sauter ». Vale a dire, la ricostruzione ex post è soprattutto intesa a servire la progettazione ex ante. E questa è l'avvertenza da tenere ben presente nel cercare il filo dirimente tra filosofia e scienza.

\section{Ricerca e applicabilità}

Se la filosofia genera un sapere scientifico che finisce per ripudiarla, ci deve pur essere, nel filosofare, una mancanza o una insufficienza costitutiva, e cioè un vuoto che nessun filosofare, in nessuna delle sue tantissime varietà, riesce a colmare. Qual è questo vuoto? Se si considera che la scienza attende a « trasformare » la realtà, a dominarla con l'azione - intervenendo - e non soltanto con il pensiero, la risposta viene da sé: la filosofia difetta di operatività o, detto piú semplicemente, di applicabilità.

14 Come esempio di teoria filosofica vedi, da ultimo, B. de Jouvenel, The Pure Theory of Politics, Cambridge, Cambridge University Press, 1963; e come esempio di teoria empirica, C. J. Friedrich, Man and bis Government, New York, McGraw-Hill, 1963. 
Non si dà scienza senza teoria. Ma la scienza - a differenza della filosofia - non è soltanto teoria. La scienza è teoria che rinvia alla ricerca, e ricerca (esperimento, o comunque acquisizione di dati) che riopera sulla teoria. Non è tutto: la scienza è anche applicazione, traduzione della teoria in pratica ${ }^{15}$. E vero che il dibattito metodologico delle scienze sociali ha investito soprattutto il rapporto tra teoria e ricerca, lasciando in penombra il rapporto tra teoria e pratica (o prassi). Ma basta allungare lo sguardo alla piú avanzata delle scienze dell'uomo l'economia - per avvertire che la scienza non è teoria che si esaurisce nella ricerca, ma anche teoria che si prolunga nella attuazione pratica: un progettare per intervenire, una prasseologia ${ }^{16}$.

Sono dunque due gli elementi che la scienza, differenziandosene, aggiunge o sostituisce al filosofare: 1) la ricerca come strumento di convalida e di fabbricazione della teoria, 2) la dimensione operativa, e cioè la traducibilità della teoria in pratica. Non occorre soffermarsi sul rapporto, o meglio sulla circolarità, tra teoria e ricerca. E invece importante chiarire, in ordine al rapporto tra teoria e pratica, la nozione di operatività o di applicabilità. Una teoria operativa, o applicabile, è una teoria che si traduce in pratica in modo conforme, e cioè come previsto e stabilito dal disegno teorico. Per applicabilità si deve intendere, allora, la corrispondenza dell'esito al proposito, del risultato alla previsione. In parole povere, l'applicabilità è l'applicazione che « riesce », non l'applicazione che fallisce producendo risultati non previsti e non voluti.

La filosofia non è, dunque, un pensare per applicare, un pen-

15 Sullo sviluppo della ricerca vedi H. Alpert, The Growth of Social Research in the United States, nel vol., a cura di D. Lerner, The Human Meaning of the Social Sciences, New York, Peter Smith, 1959. Nello stesso vol. si esamina anche il rapporto con l'azione (M. F. Millikan, The Relation of Knowledge to Action), e si valuta la capacità di intervento dell'economista (P. A. Samuelson, What Economists Know). Ma vedi spec. A. Ranney (ed.), Political Science and Public Policy, Chicago, Markham, 1968; J. M. e W. C. Mitchell, Political Analysis and Public Policy, Chicago, Rand McNally, 1969; e anche H. D. Lasswell, The Decision Process, College Park, University of Maryland, 1965. Si parla anche di « operations research » intesa come una speciale branca del sapere applicativo: cf. M. Sasieni, A. Yaspan e L. Friedman, Operations Researcb: Methods and Problems, New York, Wiley, 1959.

${ }_{16}$ Cosí, segnatamente, L. von Mises, Human Action: A Treatise on Economics, Chicago, Regnery, 1966 (3.a ed. riv.). 
sare in funzione della traducibilità dell'idea nel fatto, e quindi proporzionato e proiettato verso l'attuazione. Come fare? Questo non è l'interrogativo del filosofo, o comunque non è l'interrogativo al quale egli sa rispondere. Se guardiamo alla filosofia, e in particolare alla filosofia politica, in chiave di programma di azione, essa risulta un programma inapplicabile. Non perché da millenni l'uomo non abbia tentato di applicare alla sua città dei programmi di derivazione speculativa. Ma perché da Platone a Marx questi «programmi filosofici » sono falliti: il loro esito non è stato quello previsto e desiderato.

Questa tesi è esposta a una obiezione specifica e ad una perplessità generale. L'obiezione specifica si ricollega a Marx che teorizzò il «filosofo rivoluzionario » inteso non a comprendere ma a cambiare il mondo - ed al marxismo inteso come filosofia della prassi, e piú precisamente della «prassi rovesciante ». Come vedremo, questa obiezione non è calzante. Resta la perplessità piú generale, in ordine alla quale si osserva che la tesi della inapplicabilità del filosofare vale per le filosofie ad alto livello astraente - propriamente metafisiche - e magari per le filosofie fortemente razionalistiche; ma che non vale per le filosofie a basso livello astraente - le filosofie empiristiche - e specialmente per il pragmatismo, per quel filosofare che teorizza la dipendenza del pensiero dall'azione e anzi fa dell'applicazione la prova, o riprova, della verità. Chi sostiene la non convertibilità pratica del filosofare ha buon gioco finché si riferisce - oggi - a Hegel e ai suoi discendenti di destra o di sinistra. Ma si prenda Aristotile o, tra i moderni, un Bentham, un John Stuart Mill, magari Spencer, e infine John Dewey. In questi casi, come la mettiamo?

Questa perplessità ripropone la consueta difficoltà: la grandissima varietà e sregolatezza del filosofare. Vi sono filosofie di tutte le specie, a tutti i livelli: e non si può generalizzare, non si può parlare di filosofia in blocco, a meno di non trovare un minimo comun denominatore che le accomuna tutte. $\mathrm{E}$ questo è, davvero, il punto.

\section{Lo spartiacque linguistico}

Filosofi e scienziati non si intendono: il linguaggio dei primi risulta incomprensibile o inutilizzabile ai secondi cosí come, viceversa, il linguaggio dello scienziato risulta oscuro o comun- 
que triviale al filosofo. E vero che anche nelle scienze, o tra le scienze, si comunica poco e male. $\mathrm{Ma}$ in quest'ultimo caso la ragione è chiara: ogni scienza crea un proprio linguaggio specializzato che risulta comprensibile, per ciò stesso, ai soli iniziati. Non è chiara, invece, la ragione per la quale il filosofo e lo scienziato non si capiscono e comunicano a stento anche quando adoperano gli stessi vocaboli.

Ripartiamo dalla considerazione che il sapere scientifico trova la propria ragione d'essere distintiva nel porsi come un sapere applicabile, come un « conoscere per intervenire». Non è una impresa da poco. E l'impresa non può riuscire senza gambe idonee. Fuor di metafora, ogni sapere passa attraverso lo strumento di un linguaggio ad boc, di un linguaggio atto a «servire » gli obiettivi di quel sapere. Occorre fissare l'attenzione, pertanto, sullo strumento linguistico. E questo - lo strumento linguistico - mi sembra essere il manico che stiamo cercando.

Quando si è detto tutto, resta da dire che filosofia e scienza sono usi linguistici diversi che si divaricano in funzione dei loro rispettivi interrogativi di fondo. L'interrogativo perenne del filosofo si riassume in un perché: beninteso un «perché » ultimo, metafisico o metafenomenico che investe la ratio essendi. Per converso l'interrogativo prioritario dello scienziato si riassume in un come. $\mathrm{Va}$ da sé che nel perché del filosofo è ricompreso un come; e, viceversa, che nel come dello scienziato è sottinteso un perché. Non è che la filosofia «spieghi »e che la scienza « descriva ». E che in filosofia la spiegazione subordina la descrizione, laddove nella scienza è la descrizione che condiziona la spiegazione. Ogni sapere «spiega ». La differenza è posta dalla ricerca. La spiegazione filosofica non accerta i fatti: li travalica e li trasfigura; la spiegazione scientifica, che presuppone la ricerca, emerge dai fatti e li raffigura. In questo senso la filosofia può essere caratterizzata come un « capire ideando », laddove la scienza risulta, caratteristicamente, un «capire osservando ». Ne consegue che la filosofia è, tendenzialmente, un « capire giustificante », una spiegazione data dalla giustificazione; laddove la scienza è un «capire causale», una spiegazione in termini di causazione.

Un primo riflesso di questa divaricazione di fondo si coglie nella diversa distribuzione - tra filosofia e scienza - del conceptum rispetto al perceptum. Nel vocabolario del filosofo prevale il concepere nel senso che non vi si presta grande attenzione al percipere, all'affinamento dei termini osservativi; lad- 
dove la scienza richiede e sviluppa un meticoloso vocabolario osservativo-percettivo. Beninteso il percipere della scienza non è una mera lettura, e non deve far pensare a una immediatezza sensistica. Il perceptum non viene prima, ma dopo il conceptum. Prima concepiamo, e poi passiamo il «concepito » al vaglio del ridimensionamento e dell'aderenza osservativa. Non a caso la filosofia della natura precede le scienze della natura, cosí come la filosofia politica precede la scienza politica.

Questo spostamento dal conceptum al perceptum viene evidenziato, e diventa massiccio, quando una scienza entra nella fase delle cosiddette definizioni operazionali, e cioè tende a definire i propri termini in funzione delle «operazioni » che ne consentono la verifica empirica ${ }^{17}$. L'operazionismo - si badi bene - è un requisito che inerisce al rapporto tra teoria e ricerca, e dunque non è l'operatività che investe il rapporto tra teoria e pratica. Ma è chiaro che una definizione operazionale spiana o comunque facilita la via alla applicabilità, alla conversione della teoria in intervento.

Quel che rende la filosofia e la scienza necessariamente diverse è dunque, in ultima analisi, la diversità del rispettivo strumento linguistico. Potremmo anche dire che la filosofia è tale perché si fonda su un uso meta-empirico del linguaggio nel quale le parole tendono ad assumere - per dirla con Croce un significato « ultra-rappresentativo ». Significato ultra o metarappresentativo che fonda la prevalenza del conceptum e che investe, appunto, un mundus intelligibilis di cui cerca il «senso », l'« essenza » e la giustificazione ultima.

Per converso, la scienza sviluppa un vasto vocabolario denotativo, e cioè osservativo-descrittivo, nel quale le parole significano quel che rappresentano. Donde la prevalenza del perceptum, di un capire descrivendo che investe, appunto, un mundus sensibilis di cui cerca le regole di funzionamento. Il che lascia anche intendere come mai il problema dell'applicabilità non si

${ }_{17}$ L'operazionismo risale a P. W. Bridgman, The Logic of Modern Physics, New York, Macmillan, 1927. Nella sua applicazione alle scienze sociali si intende per operazionismo il tentativo di collegare un concetto con le sue proprietà osservabili. A rigore, i concetti le cui operazioni non sono «pubbliche » $\mathrm{e}$ 《ripetibili » non sarebbero concetti scientifici; ma a tanto rigore non arrivano nemmeno le scienze piú avanzate. Per un riproporzionamento cf. G. Bergmann, Philosophy of Science, Madison, University of Wisconsin Press, 1957. Vedi anche C. G. Hempel, Aspects of Scientific Explanation, New York, Free Press, 1965. 
risolve in sede di filosofia ma in sede di scienza. Per intervenire sulla realtà occorre sapere come $\grave{e}$. E per accertare come è occorre un linguaggio osservativo, piegato a finalità descrittive e di rilevazione empirica, e cioè un uso linguistico nel quale le parole «stanno per » quel che rappresentano. Eे questo uso descrittivo-percettivo del linguaggio che lo rende idoneo alla conversione della teoria in pratica ${ }^{18}$.

Il principio di differenziazione qui proposto non ha presieduto alla costruzione dello scibile: è una «ricostruzione » di quella costruzione. Una ricostruzione che diventa tanto piú utile quanto piú la costruzione si complica; e che certo non giova far retrocedere sino ai greci. Non ha molto senso spartire tra filosofia e no finché l'albero del sapere è soltanto un tronco. Per contro la partizione diventa tanto piú pertinente quanto piú l'albero del sapere si sviluppa e diversifica per molteplici rami. Se non ha molto senso classificare Aristotile, ne ha classificare Rousseau. E non è affatto futile discutere se Marx fosse filosofo o no, e se egli sia davvero riuscito a liberarsi della filosofia hegeliana diventando un sociologo e un economista. Del che personalmente dubito.

Marx teorizza, si diceva, il filosofo rivoluzionario in ordine a una « unità dialettica » tra teoria e prassi qualificata dall'idea della prassi rovesciante ${ }^{19}$. Ma anche il pragmatismo argomenta che è vero in teoria soltanto quel che è vero in pratica. Cosí come Kant aveva sostenuto, all'inverso, che quel che è vero in teoria deve essere vero anche in pratica ${ }^{20}$. Sí; ma lo è davvero? Una cosa è teorizzare il fare, e tutt'altra cosa è saper fare. Una cosa è teorizzare l'unità dialettica tra teoria e prassi, e

18 Non esiste alcun studio onomatologico sistematico in ordine all'uso linguistico considerato. Se ne ha la riprova a contrario dalla rassegna di R. Jacobson, Linguistics, nel vol. coll. Main Trends of Research in the Social and Human Sciences, Mouton/Unesco, Paris e The Hague, 1970, cap. VI.

19 Per il filosofo rivoluzionario di Marx e la sua umwälzende praxis si deve far capo alle Tesi su Feuerbach del 1845. Sul punto della dialettica, cfr. N. Bobbio, La dialettica di Marx, (1958), accolto nel vol. Da Hobbes a Marx, Napoli, Morano, 1965; M. Rossi, Marx e la dialettica begeliana, Roma, Editori Riuniti, 1962-63, 2 voll.; M. Dal Pra, La dialettica in Marx, Bari, Laterza, 1965.

${ }^{20}$ Intorno al detto comune: questo può essere giusto in teoria ma non vale per la pratica (1773). La tesi di Kant è riferita alla teoria fondata sul concetto di dovere, e cioè alla teoria morale. Non è, dunque, la tesi dell'idealismo. 
tutt'altra cosa è attuarla. La prova dell'applicabilità sta nei fatti. Se una teoria è fattibile lo deve dimostrare nel suo farsi. E il farsi del marxismo ha mostrato, da mezzo secolo a questa parte, non l'unità ma la disunità tra teoria e prassi: chè la prassi si rovescia, puntualmente, come non dovrebbe, come la teoria non prevedeva e non voleva.

L'inapplicabilità della filosofia della prassi può sorprendere solo chi non si pone, e non sa porsi, sul terreno operativo. Non è che la città di Marx non si realizza perché la sua teoria è mal applicata o resta inapplicata: è, invece, che la sua teoria non è, costitutivamente, una teoria intesa ad affrontare e capace di risolvere problemi di attuazione. Non lo è in linea di fatto, perché sta di fatto che il marxismo è tutto fini e niente mezzi, tutto prescrizione e niente strumentazione, tutto esortazione e niente ingegneria. $\mathrm{E}$ non lo è, in via pregiudiziale, per questa ragione: che il linguaggio di Marx resta sino all'ultimo (a dispetto delle sue intenzioni) un linguaggio meta-empirico e metaosservativo, un linguaggio caratterizzato dallo « sforzo del concetto » al quale Hegel aveva addestrato i suoi discepoli, consenzienti o ribelli che fossero. Lo Stato di cui Marx vaticinava la scomparsa non è lo Stato di cui parlano i politologi; il suo valore-lavoro non è il valore di cui parlano gli economisti; la sua nozione di classe non è traducibile nella stratificazione sociale rilevata dai sociologi. E cosí via. Il marxismo vorrebbe essere una filosofia della prassi; ma al collaudo storico risulta per quel che è: una filosofia senza prassi, una teoria senza attuazione. Se c'è un esempio macroscopico della costitutiva inapplicabilità del filosofare, questo esempio è proprio il marxismo. Il « filosofo rivoluzionario » può sí scatenare una rivoluzione; ma nella misura in cui ne viene travolto la sua vicenda ribadisce la distanza che passa tra teoria del fare e fattibilità.

Passiamo alla obiezione generale: che la tesi della inapplicabilità del filosofare si dimostra agevolmente per le filosofie ad alta rarefazione astraente, quali l'idealismo e $\mathrm{i}$ suoi derivati (non solo il marxismo ma anche l'esistenzialismo); ma che diventa difficile da dimostrare per l'empirismo filosofico e per tutte le filosofie a basso livello di astrazione.

Conveniamone subito: il salto, o la discontinuità, tra filosofia empirica e scienza empirica è senza dubbio minore. Eppure la discontinuità resta: resta perché la trasformazione del linguaggio - sia quella operazionale come quella operativa - è impresa di lunga lena, e non si compie finché non ci poniamo 
i problemi della ricerca e gli interrogativi che sono propri del « capire per operare ». Si prenda una filosofia terra terra, quale è quella di Bentham e degli utilitaristi. Non è difficile dimostrare che dalla premesse filosofiche dell'utilitarismo si possono ricavare i piú opposti programmi di azione politica ${ }^{21}$. Lo stesso vale per il darwinismo politico di Herbert Spencer. E il punto è che l'empiria non è, di per sé, applicabilità. Un livello empirico di conoscenza facilita, o avvicina, la conversione del pensiero in azione; ma un sapere empirico non è, per ciò stesso, un sapere operativo. Quando Leon Battista Alberti discorreva della « masserizia » il suo livello di discorso era senza dubbio empirico; ma egli non preludeva per questo alla scienza dell'economia.

Non mi si fraintenda: la tesi della non deducibilità della politica dalla filosofia non costituisce in alcun modo una preclusione nei confronti della filosofia come tale. Quel che critico è solo l'abuso e il cattivo uso del filosofare: e soprattutto l'errore di chi si addestra - ai fini di una ingegneria della storia - su testi nei quali non trova quel che dovrebbe cercare, e si inganna su quel che trova. Il filosofo come tale non merita alcun rimprovero; salvo il rimprovero di non fare piú il filosofo. In una età della scienza anche la filosofia è chiamata a fare un esame di coscienza: ma per ritrovare se stessa, e riprendere la propria strada. Che non è quella di reduplicare la scienza, di esserne soltanto il momento maieutico e metodologico ${ }^{22}$. Ridurre la filosofia alla epistemologia della fisica, o altrimenti limitarla alla analisi del linguaggio, è scambiare una parte per il tutto. $\mathrm{La}$ filosofia viene accusata di essere un « sapere infecondo ». $\mathrm{Ma}$ chi accetta questa critica è davvero vittima di un complesso della scienza. Non è solo che il sapere fecondo lievita tra le pieghe di quello dichiarato infecondo. E che la filosofia crea

21 Sulla non convertibilità dell'utilitarismo filosofico nella politica degli utilitaristi, vedi J. C. Rees, Le relazioni tra teoria e pratica politica, in «Il Politico», XXIV (1959), pp. 213-233.

22 In Italia la tesi della filosofia politica come metodologia della scienza politica è sostenuta, per es., da R. Treves, Intorno alla nozione di flosofia politica, in "Rivista di Filosofia», L (1959), n. 3. Vedine anche La notion de philosopbie politique dans la philosopbie italienne, nel vol. coll. cit., L'idée de philosopbie politique. Ma vedi contra A. Passerin d'Entrèves, La filosofia politica, nella Storia delle idee politiche, economiche e sociali, diretta da Luigi Firpo, Torino, UTET, 1972, vol. 6; e la discussione al riguardo nel vol. coll. cit., Tradizione e novità della filosofia della politica. 
le idee, crea i valori: e non saprei pensare a fecondità maggiore. Con questo è anche detto che la tesi della inapplicabilità del filosofare non deve essere scambiata per una sottovalutazione dell'« efficacia pratica » del filosofare. Sarebbe assurdo, visto che è la filosofia che elabora le visioni del mondo.

Segnare i confini del filosofare è anche, al tempo stesso, delimitare la scienza. Di tanto il filosofo non può surrogare lo scienziato, di altrettanto l'uomo di scienza non può soppiantare il filosofo. Con questo non vorrei che la mia insistenza sul rapporto scienza-pratica venisse fraintesa. Dire che la scienza nasce dall'esigenza di osservare una realtà sulla quale si vuole « operare » non equivale a sposare una visione grettamente praticistica della scienza. La scienza è pregiudizialmente scienza «pura» che serve uno scopo scientifico: e dunque lo scopo scientifico non è, di per sé, uno scopo pratico. Il che non toglie che lo scopo scientifico e lo scopo pratico stanno tra di loro - a dispetto delle frizioni contingenti - come due linee destinate a convergere. Basti considerare che l'applicazione è il sostituto dell'esperimento in quelle scienze che sperimentali non sono.

Ricapitoliamo. Ho sostenuto che qualsiasi filosofare trova il suo minimo comun denominatore in un linguaggio meta-osservativo inteso a «spiegare ideando », plasmato dal concipere assai piú che dal percipere. Dal che consegue che il sapere filosofico si differenzia sempre dal conoscere scientifico quantomeno a questo titolo: per una strumentazione linguistica che non soddisfa il requisito operazionale (la ricerca) e tantomeno le esigenze operative. Pertanto la scienza è caratterizzata da una applicabilità che la filosofia non possiede. Ovviamente questo fundamentum divisionis indica delle linee di tendenza, delle prevalenze. Trattandosi di una ricostruzione ex post, essa non riflette una divisione di obiettivi e di competenze scientemente perseguita dagli interessati. Ma perciò non vale ribattere che filosofia e scienza - per come sono qui divise - si trovano sovente mescolate. Appagarsi di questa constatazione equivale a santificare il passato ed a perpetuarne gli errori. Sarà verissimo che la letteratura abbonda di ibridi, e che noi continuiamo a prospettare soluzioni filosofiche di problemi pratici. Ma se riteniamo che questo sia un errore, allora occorre separare i due elementi e trovare un criterio di ricostruzione valevole per la prospezione, per dividere in avvenire quel che abbiamo pasticciato in passato. 


\section{Scientificità e avalutatività}

Eravamo restati alla scienza politica in senso lato: un modo autonomo di studiare la politica nella sua autonomia. Una accezione che si precisa mano a mano che avviene il distacco dalla filosofia politica. $\mathrm{Ma}$ a questo modo vediamo la scienza politica dall'esterno, soprattutto per cosa non è. Vediamola ora dall'interno, per come si viene facendo e «scientificizzando ${ }^{23}$. E cioè veniamo al passaggio dalla accezione lata alla accezione stretta della disciplina.

Le fasi e gli aspetti del procedimento scientifico sono molteplici. Alcune sono comuni a tutte le scienze; altre non lo sono. Un elemento comune, pregiudiziale, e sul quale non si insiste mai abbastanza, è l'elaborazione del linguaggio. A questo riguardo la regola generale è che ogni scienza si pone, ad un tempo, come: 1) un linguaggio consapevole che viene costruito riflettendo sulla propria strumentalità, 2) un linguaggio critico nel senso che nasce per correzione dei difetti del linguaggio comune o ordinario, 3 ) un linguaggio specializzato che sviluppa un vocabolario tecnico ed esoterico, 4) un linguaggio che consenta la cumulabilità e la ripetibilità. In concreto, una conoscenza del tipo «scienza » richiede e presuppone queste operazioni onomatologiche: primo, la definizione, e per essa anche la stabilizzazione (relativa) dei propri concetti portanti; secondo, la creazione di nuove parole al fine di disporre di un vocabolario adeguatamente preciso e articolato; terzo, l'adozione di una precisa sintassi logica.

Fermati i requisiti onomatologici, i vari passi e momenti del procedimento scientifico si possono riassumere cosí: a) costruzione di concetti empirici; b) costruzione di classificazioni e tassonomie; c) formulazione di generalizzazioni e di leggi di tendenza, di regolarità, o probabilistiche; d) teoria intesa come insieme di generalizzazioni interconnesse, come schema concet-

${ }^{23}$ Sulla applicazione del procedimento scientifico alla scienza politica, cf. ult. A. C. Isaak, Scope and Methods of Political Science: An Introduction to the Methodology of Political Enquiry, Homewood, Dorsey, 1969; e R. T. Golembienwski, W. A. Welsh e W. J. Crotty, A Methodological Primer for Political Scientists, Chicago, Rand McNally, 1969. Vedi anche E. J. Meehan, The Theory and Method of Political Analysis, Homewood, Dorsey, 1969. Sulla nozione di scienza in generale è utile la ricapitolazione di W. Gee, Social Science Research Methods, New York, Appleton Century Crofts, 1950. 
tuale ordinatore e unificante. In sostanza, all'inizio prevale il momento della rilevazione descrittiva (la fase classificatoria di ogni scienza), al quale segue il momento della spiegazione causale e della sistemazione teoretica. Nel complesso la scienza si configura come una «spiegazione empirica » fondata sulla rilevazione dei fatti, intesa ad arrivare a «previsioni » del tipo se-allora che ne costituiscono, ad un tempo, la verifica e la dimensione operativa.

Fin qui i requisiti comuni - piú o meno adeguatamente soddisfatti - di ogni conoscere che si dichiari scientifico. Dove le scienze si dividono - per forza maggiore - è sui procedimenti e sulle tecniche di controllo. Un sapere scientifico non è tale se le sue ipotesi e generalizzazioni non sono verificabili (o falsificabili), e cioè se non sono controllabili. In linea di principio è chiaro che tutte le scienze sono egualmente interessate a tutti i possibili modi di controllo. In linea di fatto ogni scienza si deve contentare dei controlli che può fare. Non a caso la partizione piú fondamentale è tra scienze sperimentali e no, e cioè tra le scienze che possono o non possono utilizzare il controllo dell'esperimento.

Grosso modo, la verifica o falsificazione delle asserzioni di fatto può avvenire in quattro modi: l'esperimento, il controllo statistico, il controllo comparato, il controllo storico. E superfluo spiegare perché il metodo di controllo piú forte - l'esperimento - è pressoché inaccessibile per le scienze dell'uomo, eccezione fatta per la psicologia. Il controllo statistico è largamente utilizzato in economia e, seppure in misura assai minore, in sociologia. Anche la scienza politica ricorre, quando può, al trattamento statistico: ma i dati quantificati di cui dispone sono spesso insufficienti, ancor piú spesso triviali, e sovente di dubbia validità. $\mathrm{Ne}$ consegue che il piú delle volte il politologo non ha scelta: deve ricorrere al controllo comparato e, in subordinata ipotesi, al controllo storico (che è, in sostanza, una comparazione longitudinale o diacronica) ${ }^{24}$.

24 Sul controllo e metodo comparato, cf. il vol. coll. a cura di R. T. Holt e J. E. Turner, The Methodology of Comparative Research, New York, Free Press, 1970; G. Sartori, La politica comparata: premesse e problemi, e A. Lijphart, Il metodo della comparazione, entrambi in « Rivista Italiana di Scienza Politica », I (1971), pp. 7-66 e 67-92. Vedi anche la Introduzione di G. Urbani alla Antologia di politica comparata da lui curata, Bologna, Il Mulino, di prossima pubblicazione. Sul controllo storico cf. R. Jensen, History and the Political Scientist, nel vol. coll. 
Nell'ambito di queste premesse, quand'è che si affaccia una scienza politica in senso stretto, tale da porre la distinzione tra una fase pre-scientifica della disciplina e la sua fase propriamente scientifica? La svolta avviene a cavallo degli anni cinquanta, in funzione della cosiddetta « rivoluzione behaviorista ». Naturalmente questa rivoluzione incubava da tempo. L'introduzione delle tecniche quantitative risale a Stuart Rice e a Harold Gosnell, cosí come molte premesse del nuovo corso erano state poste, tra il 1908 e il 1930, da Bentley, Merriam, e Lasswell ${ }^{25}$. Ma non si può parlare di una svolta della disciplina nel suo complesso sino a dopo la seconda guerra mondiale ${ }^{26}$.

A detta di David Easton il comportamentismo (behavioralism) modifica la scienza politica tradizionale per ben otto rispetti. Tra questi: 1) la ricerca di regolarità e di uniformità, 2) la subordinazione di ogni asserzione alla verifica empirica, 3) l'adozione di precisi metodi e tecniche di ricerca, 4) la quantificazione, 5) la avalutatività ${ }^{27}$. Detto in breve, la rivoluzione behaviorista è l'applicazione effettiva del « metodo scientifico » allo

curato da S. M. Lipset, Politics and the Social Sciences, New York, Oxford University Press, 1969; S. L. Thrupp, Diacronic Methods in Comparative Politics, nel vol. The Methodology of Comparative Research, cit.; e segnatamente l'ultimo Almond, Strategie per lo studio dello sviluppo politico, in « Rivista Italiana di Scienza politica », I (1971), spec. pp. 24856 , ove si illustra la trasformazione degli episodi storici in « episodi analitici ». Un altro tipo di trattamento è quello delle « sequenze », ora da vedere in AA.VV., Crises and Sequences in Political Development, Princeton, Princeton University Press, 1971, spec. il cap. di Sidney Verba. Cfr., anche, R.A. Dahl, Polyarcby, New Haven, Yale University Press, 1971 , cap. 3.

${ }^{25}$ I testi rilevanti dei precursori cit. sono: A. Bentley, The Process of Government, Chicago, University of Chicago Press, 1908; C. E. Merriam, New Aspects of Politics, Chicago, University of Chicago Press, 1925; e H. D. Lasswell, Politics: Who Gets What, When, How, New York, McGraw-Hill, 1936.

26 E, questa, la svolta di tutte le scienze sociali. Per la distanza che separa l'inizio degli anni cinquanta da quello degli anni settanta, è istruttivo il confronto tra due volumi panoramici commissionati e pubblicati dall'UNESCO: Contemporary Political Science, del 1950, e i capp. di Lazarsfeld, W. J. M. Mackenzie e Rokkan nel vol. cit. del 1970, Main Trends of Research in the Social and Human Sciences.

27 The Current Meaning of 'Behavioralism', nel vol, coll. a cura di J. C. Charlesworth, Contemporary Political Analysis, New York, The Free Press, 1967; tr. it. Teorie e metodi in scienza politica, Bologna, Il Mulino, 1971 pp. 52-53. 
studio della politica ${ }^{28}$. Le caratteristiche distintive di questa scientificizzazione si colgono soprattutto in tre sviluppi: la ricerca, la quantificazione, la matematizzazione.

Come già notavo, è troppo presto per dire se questa volta l'incontro tra matematica e politica sarà fecondo, e fino a che punto lo sarà. Importa avvertire, peraltro, che in questo sviluppo non c'è soltanto l'adozione di un modello o paradigma « fisicalista »; c'è anche l'influenza degli economisti, e soprattutto l'esigenza di introdurre nella disciplina una rigorosa $\mathrm{e}$ precisa sintassi logica, e cioè il «potere deduttivo » che è proprio della formalizzazione matematica. E mentre l'adozione del modello fisicalista si presta all'accusa di indebito perfezionismo, non c'è dubbio che la scienza politica ha molto da imparare dallo sviluppo matematico dell'economia e che il rigore logico di un addestramento matematico costituisce una acquisizione positiva ${ }^{29}$.

In merito alla quantificazione, misurazione, e per essa al trattamento statistico dei dati, il problema non è se la scienza politica debba diventare quantitativa o no. Il problema è se $\mathrm{i}$ dati quantitativi disponibili, o di cui possiamo ragionevolmente prevedere l'acquisibilità, siano « rilevanti » ai fini dei problemi che il politologo si pone. Nessuno contesta che una misurazione è meglio di una stima impressionistica, fatta a naso. Quel che si contesta è che la scienza politica possa essere ricondotta, e ridotta, all'àmbito del quantificabile. Nella misura in cui è la natura dei dati (se quantitativi o no) che stabilisce

${ }^{28}$ Vedi, in generale, D. B. 'Truman, The Impact on Political Science of the Revolution in the Behavioral Sciences, in AA.VV., Research Frontiers in Politics and Government, Washington D. C., Brookings, 1955; H. Eulau, S. J. Eldersveld e M. Janowitz (eds.), Political Behavior: A Reader in Theory and Research, Glencoe, Free Press, 1956; R. A. Dahl, The Behavioral Approach in Political Science: Epitaph for a Monument to a Successful Protest, tr. it. in Antologia di scienza politica, a cura di G. Sartori, Bologna, Il Mulino, 1970, pp. 65-73; H. Eulau (ed.), Political Behavior in America: New Directions, New York, Random House, 1966; i contributi di Easton, Eulau e M. Q. Sibley, in Teorie e metodi in scienza politica, cit.; B. Berelson, voce Behavioral Sciences, in International Encyclopedia of the Social Sciences, cit.; H. Eulau (ed.), Bebavioralism in Political Science, New York, Atherton Press, 1969. Per una stringata monografia cfr. infine, $\mathrm{H}$. Eulau, The Behavioral Persuasion in Politics, New York, Random House, 1963.

29 Sullo stato della questione ad oggi vedi ult. R. Boudon, Mathematical Models and Methods, nel vol. coll. cit. Main Trends of Research in the Social and Human Sciences. 
quali sono i problemi, nella stessa misura la scienza politica rischia di scoprire «piú e piú » in merito a « meno e meno »: di diventare precisa, anzi esatta, su cose triviali. Ben vengano, dunque, $\mathrm{i}$ dati quantitativi suscettivi di trattamento statistico; ma il fatto di essere dati esprimibili in numeri non li rende per ciò stesso importanti, e cioè non costituisce un criterio di rilevanza.

Lo sviluppo indiscusso e centrale della rivoluzione behaviorista è dunque quello indicato per primo: la ricerca, e per essa la saldatura tra lavoro al tavolino e lavoro sul terreno. E qui che il behaviorismo lascia la sua impronta decisiva. La ricerca non è soltanto acquisizione di dati, quantificabili o qualitativi che siano, e quindi acquisizione di nuove informazioni e di nuovi elementi di verifica. La ricerca modifica, in primo luogo, la natura dell'informazione, che non è piú fornita dall'esperienza storica ma dall'osservazione diretta, dall'indagine sul campo. In secondo luogo la ricerca finisce per trasformare, sia pure inavvertitamente, il linguaggio. La ricerca richiede che $\mathrm{i}$ concetti vengano ricondotti alle loro proprietà osservabili, e cioè richiede definizioni operazionali. È questa operazionalizzazione che ci dice che cosa possiamo e dobbiamo cercare. E dunque $\mathrm{i}$ frutti della ricerca non sono da contabilizzare soltanto in sede di informazione, ma ancor piú in ordine alla creazione di un linguaggio osservativo-percettivo capace di vera aderenza empirica.

Tra i connotati distintivi della scienza politica behaviorista ho sorvolato sinora sulla Wertfreibeit, sulla « libertà dal valore »; e questo non solo perché dobbiamo attribuire a Max Weber quel che gli spetta, ma anche perché il principio della avalutatività si soppesa meglio da ultimo, dopo aver esaminato il resto ${ }^{30}$. Da almeno trent'anni a questa parte la Wertfreibeit è il grande cavallo di battaglia non solo tra filosofi e no, ma

30 Per la tesi di Weber cf. spec. i due saggi del 1904 e 1917, accolti nel vol. Il metodo delle scienze storico-sociali, Torino, Einaudi, 1958. Sul problema segnalo: G. Myrdal, Value in Social Theory - A Selection of Essays on Metbodology, New York, Harper \& Row, 1958; D. Waldo, 'Values' in the Political Science Curriculum, in R. Young (ed.), Approaches to the Study of Politics, Evanston, Northwestern University Press, 1958. Una discussione che illustra la complessità del problema è quella tra N. Bobbio, V. Scarpelli, A. Passerin d'Entrèves e F. E. Oppenheim, Libertà come fatto e come valore, in «Rivista di Filosofia », LVI (1965), pp. 335-354. 
anche all'interno delle scienze sociali. Per il primo rispetto ha segnato il confine tra la filosofia che «prescrive $i$ valori » e la scienza che « accerta $i$ fatti ». Per il secondo rispetto ha segnato il confine tra $\mathrm{i}$ tradizionalisti, tacciati di essere valutativi, e i giovani turchi del behaviorismo. E singolare notare che oggi le parti sono, a questo ultimo effetto, invertite: i behavioristi sono sotto accusa per la loro «avalutatività conservatrice », mentre la nuova sinistra predica e reclama la « libertà di valutare ${ }^{31}$.

Vale premettere che lo status logico ed epistemologico della questione è in altissimo mare. Non siamo in chiaro, in primo luogo, su cosa siano $\mathrm{i}$ 《valori »; e nemmeno sulla differenza che passa tra valori e «valutazioni ». In secondo luogo, il nesso «valori-prescrizioni » è fragile: perché non è affatto detto che una prescrizione sia sempre in funzione di una valutazione. A questo modo si confondono gli imperativi assiologici con gli imperativi tecnici, e cioè con le regole di raccordo tra mezzi e fini. In terzo luogo, resta da risolvere il nodo della Wertbeziebung, della weberiana « relazione al valore ». Posto che l'osservatore non sia valutativo, il fatto resta che gli osservati lo sono: e non solo perché «valutano », ma per il fatto stesso di usare un linguaggio imbevuto sino al midollo di connotazioni apprezzative o derogatorie, di filie o fobie. Il che pone all'osservatore il problema di come «ricevere » il linguaggio degli osservati. $\mathrm{Se}$ non lo riceve, risulterà un cattivo osservatore. Se lo riceve tal quale, recepirà un linguaggio valutativo che lo espone all'accusa di non essere wertfrei. Forse la soluzione sta nel trovare regole di trasformazione: regole che siamo lontanissimi dall'aver trovato.

Il problema è davvero intricato. A tanto maggior ragione conviene vederlo in prospettiva, nelle proporzioni che gli competono, e distinguendo tra i due casi: quello che investe la demarcazione tra filosofia e no, e quello che investe la polemica intestina tra politologi.

In ordine al rapporto tra filosofia e scienza la dicotomia tra

31 Il meglio di questa linea critica si trova in C. McCoy e J. Playford (eds.), Apolitical Politics: A Critique of Bebavioralism, New York, Crowell, 1967. Cf. anche in T. Roszak (ed.), The Dissenting Academy, New York, Pantheon, 1967, il contributo di C. Bay, The Cheerful Science of Dismal Politics, tr. it. L'università del dissenso, Torino, Einaudi, 19692. Bay è forse l'autore di maggior statura intellettuale della nuova sinistra americana. Per quella europea è di rito ricordare Habermas e, in generale, la scuola di Francoforte. 
valori e fatti non merita il rango di capofila: come tale è fuorviante. In prospettiva conviene rivederla in funzione della differenziazione in itinere tra uso meta-empirico e uso empirico del linguaggio. I valori e le connotazioni valutative sono piaccia o non piaccia alla filosofia analitica - elemento costitutivo di un linguaggio inteso a cogliere il senso della vita, l'essenza delle cose e la ragion d'essere (teleologica, non causale) del mondo: e cioè del linguaggio filosofico. Per converso - lo si voglia o no - il discorso valutativo non trova, nel linguaggio scientifico, un veicolo adatto. Un sapere inteso a scire per causas ed a spiegare descrivendo, non ha genuino potenziale assiologico: al massimo dichiara o sottintende dei valori che non sono farina del suo sacco. In questa prospettiva la divaricazione tra discorso teleologico, normativo e assiologico da un lato, e discorso eziologico e avalutativo dall'altro, si risolve - alla distanza - nella divaricazione tra linguaggio filosofico e linguaggio scientifico, in funzione di questa regola di massima: che il potenziale assiologico del linguaggio diventa tanto minore quanto maggiore ne diventa il potenziale empirico, l'aderenza osservativa. Non è un caso che il rilancio della « scienza valutante » da parte dei contestatori e della nuova sinistra si manifesti in panni filosofici o comunque di sociologie filosofizzanti, e cioè ritornando a quel linguaggio «poietico » che caratterizza da sempre il pensiero speculativo.

Veniamo alla polemica sulla Wertfreibeit che dilacera la scienza politica - e anche la sociologia - dall'interno. Qui si deve distinguere tra (almeno) due interpretazioni: la tesi di chi raccomanda la neutralizzazione, e la tesi di chi propugna la cancellazione dei valori.

La prima scuola si attesta all'incirca su queste raccomandazioni: 1) separare i giudizi di fatto dai giudizi di valore; 2) esplicitare $i$ propri valori in premessa, o altrimenti accertare e descrivere prima di valutare; 3 ) attenersi a regole di imparzialità, quale quella di presentare con equità tutti i vari punti di vista di valore. E chiaro: queste regole non eliminano i valori; si limitano a neutralizzarli. Per questa interpretazione l'importante è di non confondersi, di non scambiare il « dover essere » per l'essere, e di non contrabbandare delle preferenze di valore per dei fatti. Il che equivale a dire che valori e valutazioni non ostacolano un sapere scientifico a condizione che siano identificati per tali, che stiano al loro posto, e che non disturbino le rilevazioni descrittive. 
La seconda scuola richiede, seppure in ordine sparso e piú confusamente, qualcosa di piú e di diverso: un vero e proprio « vuoto di valore ». I valori non debbono sparire soltanto a parte subiecti, come valutazioni dell'osservatore, ma anche a parte obiecti, in sede di registrazione delle cose osservate. Alla fine si deve puntare sulla «purificazione» del linguaggio, e cioè sulla costruzione di un linguaggio asettico, di un vocabolario che espunge tutte le connotazioni di valore. L'obiezione è che a questo modo apriamo giganteschi problemi che non sappiamo risolvere. Per esempio la « caccia al valore » lascia davvero insoluto il problema della Wertbeziebung, di come l'osservatore si rapporta ai valori degli osservati. A tutt'oggi si deve anche constatare che $i$ guadagni in avalutatività posti dalla sterilizzazione del vocabolario sono pagati da perdite in precisione; lo scotto della « lingua neutra » è una minore capacità di individuazione, un minore potere discriminante. Il che si spiega, visto che il modo piú semplice di depurare un concetto è di renderlo piú « astratto » e onnicapiente. Ma a tutte queste critiche si può rispondere che un programma di difficile attuazione non è per questo un programma sbagliato, e che quel che non riesce subito può riuscire alla distanza.

Comunque sia, il punto da fermare è che le due tesi sono diverse, diversissime, e che non giova difendere $o$ attaccare la Wertfreibeit senza precisare di quale avalutatività stiamo parlando. In definitiva la prima tesi - quella della neutralizzazione dei valori - si risolve in un puro e semplice «principio regolativo $»$, in regole intese a fondare l'imparzialità $e$, in questo senso, l'oggettività della scienza. In sostanza questa Wertfreibeit pone e si pone come una etica professionale. Bobbio lo dice benissimo: « l'avalutatività è la virtú dello scienziato come l'imparzialità è la virtú del giudice ${ }^{32}$. Anche se il giudice non è mai perfettamente imparziale, non ne consegue che gli si debba raccomandare di non esserlo. Analogamente, riconoscere i limiti dell'obiettività scientifica non autorizza a teorizzare il diritto della soggettività settaria. E come misconoscere l'importanza della Wertfreibeit regolativa, e cioè di una etica professionale, per una disciplina quale la scienza politica?

La seconda tesi - quella della tabula rasa - non si pone, invece, come un principio regolativo, sibbene come un «principio costitutivo ». Lo sconquasso è grosso, e per giustificarne

32 Considerazioni sulla filosofia politica, cit., p. 377. 
i costi e le difficoltà occorre dimostrare che la purificazione del vocabolario - perché a questo si deve approdare - è condizione tassativa di scientificità. Questa Wertfreibeit si giustifica, insomma, solo se dimostriamo che è un requisito epistemologico, e anzi lo spartiacque tra quel che è, o non è, scienza. E dunque è chiaro che chi difende la prima tesi non è tenuto a difendere la seconda; cosí come il rifiuto della seconda tesi non coinvolge affatto la prima.

Concludo. In sede epistemologica mi sembra difficile sostenere che un sapere scientifico dipenda, in primissimo e determinante modo, dalla sua avalutatività. Chi eleva la Wertfreibeit a requisito primario e sine qua non della scientificità pecca di esagerazione, e anche di semplicismo. I requisiti che presiedono alla formazione di un linguaggio scientifico sono assai piú determinanti. Scienze quali la psicologia e l'economia hanno fatto la loro strada perseguendo o presupponendo - piú o meno implicitamente - fini di valore. La medicina non è danneggiata dal ritenere la salute un bene. Dal che sembra discendere che l'avalutatività è un «principio regolativo », non un principio costitutivo. Conclusione che non solo restituisce alla disputa le sue proporzioni, ma che ne chiarisce i tenmini. Mentre la neutralizzazione dei valori risulta - quantomeno per la scienza politica - un principio regolativo di fondamentale importanza, la elisione dei valori si pone come un principio costitutivo che resta da dimostrare. Chi sottoscrive la prima Wertfreibeit, non è tenuto a sottoscrivere la seconda. Ed è la seconda Wertfreibeit, assai piú e meglio della prima, che offre argomenti a chi predica una «scienza valutante » che è, insieme, cattiva filosofia e pessima scienza.

\section{Un bilancio}

Qual è il bilancio, all'inizio degli anni settanta, della scientificizzazione del politologo? I piú lamentano che la scienza politica non sia abbastanza «scienza ». Ma è piú interessante chiedere che cosa abbia guadagnato dalla propria scientificizzazione. Gli eccessi della rivoluzione behaviorista sono stati in buona parte riconosciuti e corretti dagli stessi colpevoli. Cosí la fase iperfattualista e crudamente scientista è stata largamente superata. Semmai le perplessità si appuntano sull'eccessivo tecnicismo e su un abuso di formule matematiche il cui 
fumo nasconde l'assenza di arrosto. Anche l'eccesso di operazionismo suscita fondate perplessità. Della operazionalizzazione del linguaggio si deve dire bene nella misura in cui produce quel linguaggio osservativo-percettivo senza il quale non c'è vera aderenza empirica. Ma bisogna anche rendersi conto dei limiti dell'operazionismo, del fatto che le definizioni operazionali sviluppano l'estensione o denotazione dei concetti a scàpito della loro intensione o connotazione. Dal che consegue che un operazionismo ossessivo e male inteso atrofizza la teoria, e cioè la fecondità teoretica dei concetti.

Nonostante queste riserve, e altre ancora, si può convenire con Easton: negli anni sessanta è avvenuto il passaggio dalla scienza politica « sintetica » alla scienza politica «teoretica » ${ }^{33}$. Il che sta a dimostrare che gli eccessi dell'operazionismo, della quantificazione e del matematismo non hanno impedito il parallelo sviluppo della costruzione teoretica. È vero che, teoreticamente parlando, la disciplina si trova in piena diaspora. Gli schemi concettuali e gli approcci tra $\mathrm{i}$ quali scegliere sono una pletora: dalla teoria generale dei sistemi alle teorie cibernetiche, struttural-funzionali, decisionali, stocastiche, del gruppo, dei ruoli, dei conflitti, dello sviluppo, del potere, e altre ancora; il tutto variamente specificato ed intrecciato ${ }^{34}$. Ma occorre sottolineare che questo stato di confusione riflette la nascita endo-

33 Voce Political Science, in International Encyclopedia of the Social Sciences, cit., vol. XII, p. 297. Assieme a questa voce, e a titolo di primo orientamento sullo stato della disciplina, vedi M. D. Irish (ed.), Political Science: Advance of a Discipline, Englewood Cliffs, Prentice-Hall, 1968; e W. J. M. Mackenzie, The Study of Political Science Today, Basingstoke, Macmillan, 1972. Meglio, tuttavia, la bibliografia della nota seg.

${ }_{34}$ Per le varie teorie e schemi concettuali un panorama complessivo può essere ricavato da: W. T. Bluhm, Theories of the Political System, Englewood Cliffs, Prentice-Hall, 1965; l'eccellente volume curato da Easton, Varieties of Political Theory, Englewood Cliffs, Prentice-Hall, 1966; J. C. Charlesworth (a cura di), Teorie e metodi in scienza politica, cit.; E. Meehan, Contemporary Political Thought: A Critical Study, Homewood, Dorsey Press, 1967; O. R. Young, Systems of Political Science, Englewood Cliffs, Prentice-Hall, 1968, tr. it. Prospettive d'analisi in scienza politica, Bologna, Il Mulino, 1972; S. M. Lipset (ed.), Politics and the Social Sciences, cit.; Antologia di scienza politica, a cura di G. Sartori, cit.; M. Haas e H. S. Kariel (eds.), Approaches to the Study of Political Science, Scranton, Chandler, 1970.

Per una valutazione d'insieme di due importanti settori della disciplina vedi, inoltre, G. Pasquino, Modernizzazione e sviluppo politico, Bologna, Il Mulino, 1970; e G. Urbani, L'analisi del sistema politico, Bologna, Il Mulino, 1971. 
gena della teoria, e cioè l'affermarsi di una teoria che non è piú un prestito o comunque un tertium genus, ma il frutto della riflessione che $\mathrm{i}$ politologi compiono sulle cose che accertano $\mathrm{e}$ che cercano. Difatti anche la teoria sta imparando a usare il « linguaggio delle variabili »: segno caratteristico, e caratterizzante, di una riflessione teoretica che emerge $a b$ intus. E dunque sembra giusto vedere questa molteplicità di approcci e di teorizzazioni come una crisi feconda, come una crisi di crescenza.

Ovviamente è molto difficile generalizzare. La difficoltà non sta soltanto nella fase altamente dinamica della disciplina; sta anche nel numero dei suoi cultori. Negli Stati Uniti i dipartimenti di scienza politica sono piú numerosi dei dipartimenti di sociologia, e il numero dei politologi di professione può essere stimato attorno a ottomila ${ }^{35}$. Non può stupire che siffatti numeri producano di tutto un po', in una straordinaria varietà di contenuti, di indicazioni e di direzioni. Senza contare la tradizione inglese e, in generale, dell'Europa continentale, che riflette l'impulso dato alla disciplina dal suo rinnovamento nordamericano, ma che mantiene - nel male come nel bene caratteristiche proprie ${ }^{36}$. In generale, e generalizzando, in Eu-

35 I membri individuali della Associazione Americana di Scienza Politica sono circa 14.000. Nel 1970 l'albo dei registrati alla National Science Foundation portava 6.493 docenti di Scienza politica (cf. American Science Manpower, Washington D. C., e E. M. Baker, The Political Science Profession in 1970, in P. S., suppl. della "American Political Science Review", 1, 1971). D'altra parte la stima, che dà tutte le garanzie di attendibilità, di Eulau e March è di circa 10.000 politologi (nel vol. da loro curato, Political Science, Englewood Cliffs, Prentice-Hall, 1969, p. 67). Pertanto il numero indicato nel testo è, semmai, restrittivo.

36 Manca un soddisfacente panorama della situazione europea. La rassegna di J. Barents, Political Science in Western Europe, London, Stevens, 1961, è lieve, e superatissima. Meglio, se non fossero anch'essi vecchi, i due volumi di Jean Meynaud, Introduction à la science politique, Paris, Colin 1959, e La science politique: Fondements et perspectives, Lausanne, 1960. D'altra parte non si può ricavare molto dal disordinatissimo volume di W. J. M. Mackenzie, Politics and the Social Sciences, Harmondsworth, Penguin, 1967, tr. it., La politica e le scienze sociali, Bari, Laterza, 1969, pieno di spunti felici, ma di ineguagliabile disorganicità. Per l'Italia vedi: N. Bobbio, Teoria e ricerca politica in Italia, in «Il Politico », XXVI (1961), pp. 215-233 e, dello stesso, il vol. cit. Saggi sulla scienza politica in Italia; A. Spreafico, Studi politici e scienza politica in Italia, in Annuario Politico Italiano, Milano, Comunità, 1964; G. Sartori, La scienza politica, in AA.VV., Le scienze dell'uomo e la riforma universitaria, Bari, Laterza, 1969. 
ropa la scienza politica è in ritardo in quanto non ha ancora ben acquisito la dimensione della ricerca. Inoltre la scientificizzazione è senza dubbio minore. $\mathrm{Ma}$ non è detto che quest'ultimo sia soltanto un deficit. Come passeremo a vedere tornando al quesito iniziale: che cosa la scienza politica abbia guadagnato, ad oggi, dalla sua scientificizzazione behaviorista. Perché nel guadagnare si può anche perdere.

Se i meriti e i frutti scientifici della rivoluzione behaviorista sono innegabili, l'altra faccia della medaglia è che al progredire della scienza fa da contrappeso il regredire dell'oggetto, e cioè della politica. La messa a fuoco behaviorista porta, infatti, ad una percezione «diffusa » e « orizzontale » della politica, e per essa alla diluizione e periferizzazione della politicità.

Occorre ricordare che il behaviorismo è, all'origine, un movimento interdisciplinare, e cioè il «metodo comune » di tutte le scienze dell'uomo. In primis, dunque, il behaviorismo è la scienza comune di tutte le scienze che ne adottano i precetti. Queste scienze sono, tutte, bebavioral sciences prima di essere scienza politica, sociologica, psicologica, o anche, volendo, economica di tipo comportamentista. Fin qui nulla da obiettare. I metodi e le tecniche di indagine sono, per definizione, un patrimonio interdisciplinare, e cioè un serbatoio al quale attingono tutte le scienze nella misura in cui un metodo o una tecnica risulta idonea e utilizzabile. Il problema è se, e fino a che punto, questa unità metodologica delle scienze comportamentistiche sia da intendere come un superamento della divisione del lavoro conoscitivo, e per essa delle specializzazioni disciplinari. Il che ci riporta alla vexata quaestio della unità della scienza. Questa unità risiede in una comune piattaforma metodologica di partenza, oppure deve essere ricuperata anche al livello delle diramazioni disciplinari?

Il movimento behaviorista si dichiara interdisciplinare, non « riduzionista »; ma contiene, volente o nolente, un potenziale riduzionistico. Non c'è dubbio, per esempio, che il behaviorismo abbia contribuito alla «sociologizzazione della politica», vale a dire alla riconduzione e riduzione della scienza politica alla sociologia politica: e questo perché $\mathrm{i}$ fenomeni di cui privilegia l'osservazione sono gli stessi fenomeni osservati dal sociologo. $\mathrm{Ma}$ perciò il behaviorismo apre, sia pure di striscio, una questione di principio. Se crediamo alla soluzione riduzionista, allora non si vede perché l'argomento debba essere lasciato a questo punto: che la scienza politica è « parte » della sociologia. 
Alla stessa stregua anche l'economia è da considerare parte di un piú ampio contesto, e sarebbe quindi da ridurre alla sociologia dell'economia. Dopodiché si può passare a sostenere che anche la nozione di società è un costrutto teorico campato a mezz'aria: in realtà esistono solo « relazioni sociali », rapporti inter-soggettivi, che fanno capo a quelle unità ultime - inscomponibili, conorete e osservabili - che sono soltanto i singoli individui. Alla fine, sul filo di questa logica anche la sociologia dovrebbe scomparire, riassorbita, per esempio, nella psicologia, o comunque nella psicologia sociale. Quel che resta da dimostrare è se, a questo modo, il nostro sapere sarebbe maggiore e migliore di quello che è oggi. Del che fortemente dubito.

Con questo non si sostiene che l'attuale suddivisione disciplinare tra le scienze dell'uomo sia intoccabile. Quel che resta irreversibile - a dispetto delle facili ironie sugli steccati disciplinari - è la divisione e specializzazione del lavoro conoscitivo. La ratio di questa specializzazione può essere diversa da quella che è; ma deve essere, appunto, una ratio. $\mathrm{E}$ in attesa di trovarla importa vedere, in concreto, come funziona il «ricupero interdisciplinare » suggerito dal behaviorismo ${ }^{37}$.

Dicevo che in forza dei canoni e della prospettiva che gli è propria il behaviorismo è indotto a vedere la politica nella sua diffusione orizzontale assai piú che nella sua verticalità ${ }^{38}$. Per rendersene conto basta mettere a confronto la behaviorizzazione con la giuridicizzazione della politica, e cioè con la scuola istituzionale-legalistica che è poi, alle origini, la Staatslebre, la dottrina dello Stato. Reagendo al «legalismo » i behavioristi avevano ragione, cosí come avevano ragione nel dire che la politica non è coestensiva e tantomeno incapsulabile nell'ambito dello Stato. Ma la loro polemica va oltre il segno. Posto che le strutture formali (giuridiche) non sono le strutture reali, che cosa sono e come si identificano le strutture che interessano il politologo? Almond, e con lui il grosso dei behavioristi, defi-

${ }^{37}$ Sul behaviorismo come movimento interdisciplinare, vedi B. Berelson (ed.), The Behavioral Sciences Today, New York, Basic Books, 1963. Per la divisione e specializzazione del lavoro conoscitivo, cf. G. Sartori, Per una definizione della scienza politica, in Antologia di scienza politica, cit., spec, pp. 12-17.

${ }_{38} \mathrm{E}$ sintomatica a questo riguardo la silloge curata da Eulau, Political Behavior in America: New Directions, cit., nella quale la dimensione verticale è ricondotta e ridotta ai "gruppi »: il che rispecchia la sistematica dello stesso Eulau nel suo vol. cit., The Behavioral Persuasion in Politics. 
nisce «struttura » cosí: « attività osservabili che compongono il sistema politico »; attività che sono strutture in quanto «si verificano con una certa regolarità ${ }^{39}$. La preoccupazione comportamentista è trasparente. Ma a questo modo le strutture politiche vengono vaporizzate. In definitiva le strutture sono ricondotte a dei « ruoli ». Il che rende bene l'idea delle strutture sociali; ma non rende affatto l'idea delle strutture politiche determinate dall'ordinamento giuridico-costituzionale. L'ottica che va bene per il modo nel quale si struttura la vita sociale - la sfera degli sponte acta - non va affatto bene per il modo nel quale viene deliberatamente strutturato un sistema politico. Pertanto la scienza politica behaviorista finisce per girare intorno a qualcosa che non afferra mai: perché afferra la periferia lasciandosi sfuggire l'epicentro della politica.

Qual è allora la fetta, la grossa fetta, dei fenomeni politici che scompare dalla visuale del behaviorista di stretta osservan$z a$ ? E tutto quel che avviene nella cosiddetta « scatola nera »: nera, appunto, per chi ne resta fuori e la guarda dall'esterno. Quel che il behaviorista vede male e poco è tutto il tragitto che va dagli inputs, da quel che viene messo dentro al sistema politico, agli outputs, a quel che ne esce fuori. Nella prospettiva behaviorista questo tragitto si risolve in un processo di « trasformazione », di trasformazione degli inputs in outputs. Quel che sfugge è come il sistema politico sia anche, e talvolta soprattutto, un sistema di «produzione», di produzione di decisioni politiche. Beninteso, questi rilievi valgono per la «scienza normale ». Autori come David Easton e Karl Deutsch, o coloro che adottano la teoria dei giochi e studiano le tecniche decisionali, davvero non meritano l'accusa di aver lasciato nell'ombra la «scatola nera ». Al contrario, quel che sappiamo della meccanica del sistema politico lo abbiamo appreso soprattutto da loro. Easton, e con lui molti altri, sanno benissimo quante decisioni, spesso determinanti, sono dei witbinputs, sono messe dentro « da dentro », e cioè sono generate dalle interazioni tra quegli attori che stanno al vertice del sistema politico. Nondimeno l'idea piú recepita e avvalorata dalla scienza normale è

39 G. Almond e B. Powell, Comparative Politics: A Developmental Approach, Boston, Little Brown, 1966, tr. it., Politica comparata. Bologna, Il Mulino, 1970 , p. 59. Per una critica alla insufficienza dell'analisi struttural-funzionale di Almond, cf. il mio art., La politica comparata: premesse e problemi, cit., spec. pp. 49-62. 
che il sistema politico sia un sistema di trasformazione di inputs, non di autonoma produzione di decisioni.

Intendiamoci: non è che la « crisi di identificabilità » della politica sia tutta da addebitare al behaviorismo. Si è già ricordato che la diluizione orizzontale della politica ne riflette la massificazione, e ancor piú la democratizzazione ${ }^{40}$. Resta da ricordare che l'idea di politica diventa diffusa e evanescente anche in funzione della sua "globalizzazione », a séguito della estensione globale che caratterizza la nuova politica comparata. Nel terzo mondo e nei cosiddetti paesi in via di sviluppo troviamo società che risultano - al paragone con le nostre - società « senza Stato », vale a dire a struttura politica informe, o comunque diffusa e non specializzata. Di qui una diluizione della politica che riflette il tentativo di darne una definizione minima, tale da valere per qualsiasi aggregato umano (ivi includendo il cosiddetto sistema politico degli Eschimesi).

$\mathrm{Ma}$ se la crisi di identità della politica è il prodotto di tutta una serie di circostanze concomitanti, forse il fattore singolo di maggior peso resta il tipo di scientificità valorizzato dal behaviorismo, e per esso il fenomeno della retroazione dei dati. $\mathrm{Ci}$ occorrono dati; se sono quantificati è meglio; e se consistono di grandi numeri è ancora meglio. Orbene, il grosso dei dati di questa sorta è costituito dai dati socio-economici rilevati dagli statistici. Di qui la diffusione orizzontale e periferica della politica, che ci porta a vedere da dove nasce, a discapito di dove si coagula. Ma c'è di piú. Il punto è che se i dati ai quali facciamo capo sono dati economico-sociali, è da questi dati che dobbiamo far discendere la spiegazione. Questo tipo di informazione precostituisce l'interpretazione: da dati economico-sociali è giocoforza ricavare spiegazioni di tipo economico-sociale. Non ne consegue che $\mathrm{i}$ politologi behavioristi siano costretti a spiegare la politica con la sociologia e con l'economia. Ma certo ne consegue che la politica diventa un explanandum il cui explanans è fornito, e precostituito, da dati che potremmo dire ipo-politici, a basso tenore di politicità, e spesso di discutibile e certo indiretta rilevanza politica. E cosí la retroazione dei dati - dei dati privilegiati dal behaviorismo - ci riporta alla eteronomia della politica, alla politica spiegata $a b$ extra.

Con tutti i suoi meriti, dunque, la behaviorizzazione della scienza politica rimette in questione l'autonomia della politica.

40 Cosa è «politica», loc. cit., spec. pp. 21-26. 
Il trattamento si riflette sull'oggetto. Se la scienza è il come, il come sfuoca e, al limite, soffoca il che cosa. Donde l'accusa portata al behaviorismo di approdare, al limite, alla sparizione di ciò che è politico ${ }^{41}$. Non c'è nulla di paradossale in questo sviluppo. Al contrario è logico che una scienza politica stricto sensu, che vuol essere scienza ad ogni costo, debba tagliar fuori il «non scientificizzabile». Questa conclusione rafforza la previsione che la navigazione della scienza politica continuerà ad essere perigliosa e difficile. Di tanto la politica viene negletta - sia perché periferizzata, sia perché dichiarata eteronoma di altrettanto scappa di mano e ridiventa una forza « fuori controllo ». Ad un estremo c'è la scienza che si mangia la politica; all'altro estremo c'è la politica che si mangia la scienza. I due estremi si toccano e si convertono l'uno nell'altro. E compito del politologo impedirlo.

${ }^{41}$ L'accusa si fonda, peraltro, su una grande diversità di premesse. Cf. la distanza, da un lato, tra B. Crick, In Defence of Politics, London, Penguin, 1964, tr. it., Difesa della politica, Bologna, Il Mulino, 1969, e, dall'altro, il vol., coll. cit., Apolitical Politics: A Critique of Bebavioralism. Le critiche che piú si allineano al mio testo, meglio sviluppate nell'art., Alla ricerca della sociologia politica, in « Rassegna Italiana di Sociologia », IX (1968), pp. 597-639), sono quelle di R. Macridis, Comparative Politics and the Study of Government, in "Comparative Politics», I (1968), spec. pp. 81 e 84-87; e di G.D. Paige, The Rediscovery of Politics, in J. D. Montgomery e W. J. Siffin (eds.), Approaches to Development, New York, Mc-Graw Hill, 1966. 